\title{
UN PRÊTRE D'AMPHIPOLIS DANS LA GRANDE LISTE DES THÉARODOQUES DE DELPHES
}

"La liste des théorodoques de Delphes est un document capital pour la géographie historique des pays grecs" écrivait L. Robert il y a presque quarante-cinq ans, et il ajoutait «Si elle a été utilisée à l'occasion dans des études sur certaines parties du monde grec, elle a été plus souvent négligée, ainsi pour la Macédoine ou pour la Sicile ${ }^{1}$. Depuis, les lacunes signalées par Robert ont été comblées par G. Manganaro en ce qui concerne la Sicile $^{2}$ et par F. Papazoglou pour la Macédoine ${ }^{3}$. Dans les deux cas l'exploitation géographique de la liste a donné des résultats précieux pour la connaissance de ces régions. L'exploitation historique et prosopographique, en revanche, s'est heurtée aux incertitudes qui pesaient sur la chronologie de la liste.

A. Plassart avait daté la rédaction du corps principal de la liste des théarodoques "du premier quart du II siècle" ${ }^{4}$. En 1944/5 G. Daux, grâce à la publication d'un décret de Delphes, établit l'année 178 comme le terminus ante quem de sa rédaction ${ }^{5}$. Robert, en 1946, dans sa publication d'un nouveau fragment avèc la liste des villes d'Ionie et de Carie, acceptait pour l'essentiel la datation de Plassart, tout en proposant comme terminus ante quem l'année 188, date de la paix d'Apamée ${ }^{6}$. Cependant, trois ans plus tard, G. Daux, revint sur la question de la chronologie. Tirant parti des progrès de l'épigraphie delphique et se fondant sur des arguments aussi bien paléographiques que

(*) Nous tenons à remercier notre collègue et ami Chr. Habicht qui a bien voulu lire et corriger une première version de cet article. Nous lui sommes particulièrement redevable de nous avoir suggéré de restituer aussi bien dans la liste d'Amphipolis que dans celle de Delphes le nom de Xénotimos au lieu d'un problématique Ménotimos.

(1) L. Robert, Villes, (= L. Robert, "Villes de Carie et d'Ionie dans la liste des théorodoques de Delphes», $B C H 70$ [1946], p. 506-523), p. 506. Le grand helléniste parlait de la "grande liste" publiée par A. Plassart, "La liste des théarodoques", BCH 45 (1921), p. 1-85 - désormais Plassart, Liste.

(2) G. Manganaro, "Città di Sicilia e santuari panellenici nel III e II sec. a.C.", Historia 13 (1964), p. 414-438.

(3) F. Papazoglou, Les villes de Macédoine à l'époque romaine, BCH Suppl. XVI (1988) - désormais Papazoglou, Villes de Macédoine.

(4) Plassart, Liste, p. 41.

(5) G. Daux, "Inscriptions de Delphes", BCH 68-69 (1944-45), p. 101-103, n. 11.

(6) Robert, Villes, p. 514-515. 
prosopographiques, il suggérait que la date des trois premières colonnes, constituant le corps principal de la liste, devrait être remontée "de trente ou quarante ans" et être placée entre 230 et 220 ou 235 et 220 environ ${ }^{7}$. Le caractère inévitablement subjectif des critères paléographiques, les ambiguités des données prosopographiques, mais aussi les précautions oratoires de Daux semblent avoir laissé sceptiques les Robert, qui se contentèrent de rapporter les conclusions de leur collègue sans y souscrire ${ }^{8}$. Comme Daux aussi bien que Robert avaient annoncé, chacun de son côté, la réédition complète de la grande liste ${ }^{9}$, le monde savant resta dans l'expectative en attendant le verdict définitif des deux grands maîtres français de l'épigraphie grecque ${ }^{\mathbf{1 0}}$. La disparition récente des deux savants encouragea la réouverture du dossier ${ }^{11}$, mais la question de la date n'était toujours pas résolue. C'est la publication d'une médiocre photographie sans transcription et pratiquement sans commentaire qui vient de nous offrir une base solide pour la datation de la grande liste.

Kalliopè Lazaridou dans son rapport des fouilles du gymnase d'Amphipolis, publié en 1990 dans les Praktika de 1985 de la Société archéologique d'Athènes, mentionne à la page 74 «une inscription de marbre taillée en haut et à gauche, elle a été remployée comme chapiteau dorique (diam. 0,36 m). Elle conserve sur 11 lignes deux séries de listes d'archontes, vraisemblablement sous Philippe V (pl. 25a). Dimensions de l'inscription : $0,45 \times 0,455 \mathrm{~m}$. Elle a été trouvée en dehors de l'angle Nord-Est de la pièce [où avait été découvert] le buste d'Apellas " ${ }^{12}$. La planche 25 a permet d'établir - avec toutes réserves, vu la qualité de la photographie - le texte des cinq premières lignes et du début de la sixième.

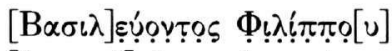

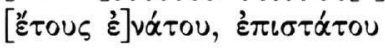

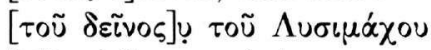

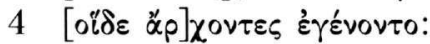

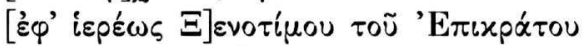

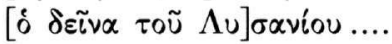

(suivent les noms d'au moins trois prêtres et d'autant d'“archontes").

Il s'agit manifestement d'une liste des archontes du gymnase, autrement dit des gymnasiarques, établie en $214 / 3$, la neuvième année du règne de Philippe $\mathrm{V}^{13}$. L'année de

(7) G. Daux, "Listes delphiques de théarodoques», REG 62 (1949), p. 21-27.

(8) BullÉpigr (1950), 127, p. 166; cf. L. Robert, «Les villes de Kéos dans la liste des théarodoques de Delphes», Hellenica XI-XII (1960), p. 167-168 et 171.

(9) G. Daux, "La grande liste delphique des théarodoques", $A J P h 101$ (1980), p. 318. Daux était revenu sur la liste dans deux autres articles : "Note sur la liste delphique des théarodoques", $B C H$ 89 (1965), p. 658-664, "Le décret de Delphes $\mathrm{SIG}^{3}$ 548", Le monde grec, Hommages à Claire Préaux (1975), p. 493 et "Trois remarques de chronologie delphique», $B C H 104$ (1980), p. 120-123. L. Robert avait annoncé son intention de rééditer la grande liste dans Villes, p. 508 ; cf. idem, Hellenica XI-XII (1960), p. 170, n. 1.

(10) Pour la Macédoine, cf. Papazoglou, Villes de Macédoine, p. 18-19; en revanche, A. Tataki, Ancient

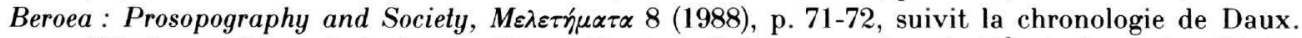

(11) La réédition de la liste a été confiée à J. Oulhen, membre de l'École française d'Athènes.

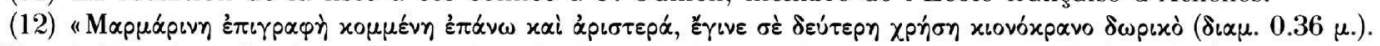

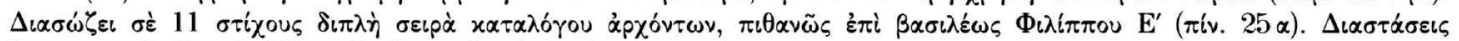

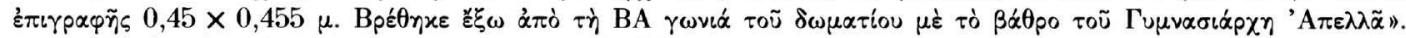

(13) Sur le calcul des années de règne de Philippe V, voir F. W. Walbank, Philip V of Macedon (1940), p. $295-299$. 
fonction de chaque gymnasiarque est datée par le prêtre d'Asklépios, qui à Amphipolis comme dans les autres cités macédoniennes était le prêtre éponyme ${ }^{14}$. Le premier de la liste s'appelle Xénotimos fils d'Épikratès. Or Plassart lisait aux lignes 78-79 de la

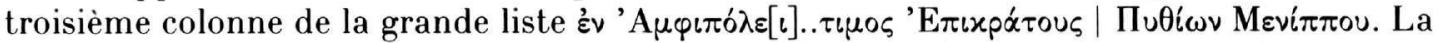
photographie et l'estampage de cette section de la stèle que nous a généreusement procurés notre collègue $\mathrm{J}$. Oulhen nous permettent de restituer dans la lacune le nom de Xénotimos ${ }^{15}$. Il n'y a pas de doute que le prêtre d'Asklépios de l'inscription d'Amphipolis et le théarodoque d'Apollon de la liste delphique ne sont qu'une seule et même personne. Une coïncidence est à exclure, d'autant plus qu'en Macédoine le culte éponyme d'Asklépios est associé à celui de son père Apollon, ce qui rend très vraisemblable que les prêtres d'Asklépios et les théarodoques de Delphes étaient recrutés dans les mêmes familles ${ }^{16}$.

L'état de la pierre ne nous permet pas de connaître la longueur de la liste. Comme nous l'avons signalé, le nom de Xénotimos était suivi de celui d'au moins trois autres prêtres ${ }^{17}$. Par conséquent, on doit considérer l'année 217/6 comme le terminus ante quem de la date de sa prêtrise, tout en soulignant que Xénotimos assuma vraisemblablement ses fonctions dans les années vingt du II $^{e}$ siècle. La nouvelle inscription ne laisse pas de doute que l'hypothèse de Daux était juste et que la rédaction du corps principal de la grande liste doit appartenir à la décennie 230-22018.

Miltiade B. Hatzopoulos.

(14) Cf. BullÉpigr (1988), 847; M. B. Hatzopoulos et L. D. Loukopoulou, Morrylos, cité de la Crestonie,

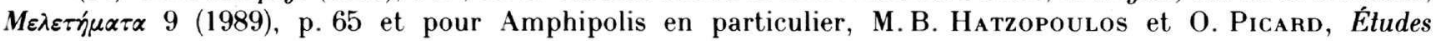
amphipolitaines, $M \varepsilon \lambda \varepsilon \tau \dot{n} \mu \alpha \tau \alpha 12$ (1991 à paraître).

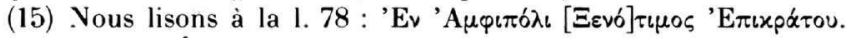

(16) Cf. BullÉpigr (1988), 847. A Kalindoia à deux reprises la prêtrise d'Asklépios est dévolue dans la mème famille: les trois frères, Ménélaos, Antigonos et Antiménon fils de Ménandros et les deux frères Kertimmas et Gydias. Nous espérons faire état prochainement d'un cas analogue à Béroia; cf. M. B. Hatzopoulos, "Un nouveau document du règne d'Antigone Gonatas", Поøxí̀ $\alpha, M \varepsilon \lambda \varepsilon \tau \dot{n} \mu \alpha \tau \alpha 10$ (1990), p. 147.

(17) Nous examinerons les autres questions que pose cette intéressante inscription dans la monographie que $\mathrm{Ph}$. Gauthier et nous-même sommes en train de préparer sur la loi sur le gymnase de Béroia.

(18) Chr. НАвіснт, Praestant interna. Festschrift V. Hansmann (1982), p. 381, avait déjà fait remarquer qu'une addition postérieure à la première rédaction de la liste (col. I 31) devait dater de $c a 200$ av. J.-C., alors que Ph. Gauthier, Nouvelles inscriptions de Sardes II (1989), p. 149-150, a fait lui aussi de son côté un rapprochement prosopographique qui milite en faveur de la datation de la "grande liste" vers 225 . 